\title{
Pacific
}

Journal of

Mathematics

\section{ANY KNOT COMPLEMENT COVERS AT MOST ONE KNOT COMPLEMENT}

ShICHENG WANG AND YING QING WU 


\title{
ANY KNOT COMPLEMENT COVERS AT MOST ONE KNOT COMPLEMENT
}

\author{
Shicheng Wang and Ying-QING Wu
}

\begin{abstract}
It follows from Culler, Gordon, Luecke and Shalen's Cyclic Surgery Theorem that any knot complement is covered by at most two knot complements. Gonzales-Acuna and Whitten proved a result on the other direction: A given knot complement can cover at most finitely many knot complements. This paper is to show that the best possible result in this direction holds: A given knot complement can nontrivially cover at most one knot complement. Moreover, if the knot is not a torus knot, then the covering map is unique up to equivalence.
\end{abstract}

Given a 3-manifold $M$, there are generically infinitely many manifolds which cover $M$. However, if we are restricted to the category of knot complements, the situation is quite different. It can be shown (see Lemma 1 and below) that if the complement $E(K)$ of a knot $K$ is $n$-fold covered by some knot complement, then the covering is cyclic, and $K$ admits a cyclic surgery, i.e. a Dehn surgery such that the fundamental group of the resulting manifold is a cyclic group $\mathbf{Z}_{n}$. It follows from the Cyclic Surgery Theorem of [CGLS] that if $K$ is not a torus knot, then there are at most two such coverings. The situation is also clear if $K$ is a torus knot: By a theorem of Moser [M], a Dehn surgery on a $(p, q)$ torus knot $T(p, q)$ is a cyclic surgery if and only if the surgery coefficient is $(k p q \pm 1) / k$ for some $k$. Now the $k p q \pm 1$ fold cyclic covering of the complement $E(K)$ of $K$ is always homeomorphic to $E(K)$ itself, with possibly an orientation reversing homeomorphism. So $E(K)$ is only covered by one knot complement, although there are infinitely many different covering maps.

In this paper we will study a closely related problem: How many knot complements are nontrivially covered by a given knot complement $E(K)$ ? The problem was studied by Gonzales-Acuna and Whitten in [GW], where they proved that a knot complement covers at most finitely many knot complements up to homeomorphism. The main result of this paper is

THEOREM 1. The complement $E(K)$ of any knot $K$ can nontrivially cover at most one knot complement $E\left(K^{\prime}\right)$ up to homeomorphism. 
REMARK 1. We say two coverings $p_{1}, p_{2}: M \rightarrow N$ are equivalent if there is a homeomorphism $h: M \rightarrow M$ such that $p_{1}=p_{2} \circ h$. If $K$ is a torus knot, then as we have seen above, there are infinitely many nonequivalent covering maps $p: E(K) \rightarrow E(K)$. In the proof of Theorem 1 we will actually show that if $K$ is not a torus knot, then the degree of the covering map $p: E(K) \rightarrow E\left(K^{\prime}\right)$ is unique. It follows that $p$ is unique up to equivalence, because a cyclic covering is determined by its degree if the first homology group of $N$ is cyclic.

REMARK 2. A generalization of Gonzales-Acuna and Whitten's Theorem was proved in [W3]: Any aspherical 3-manifold with nonempty boundary covers only finitely many 3 -manifolds.

All notations not defined in the paper are standard, see $[\mathbf{G}],[\mathbf{H}]$, [BM], [S] and [T].

Definitions. For a knot $K$ in $S^{3}, N(K)$ denotes the regular neighborhood of $K ; E(K)$ denotes the knot complement $S^{3}-\operatorname{Int} N(K)$; $(K, n / r)$ denotes the 3-manifold obtained by surgery on $K$ with coefficient $n / r$, where $n$ and $r$ are coprime integers; $L(n)$ denotes a lens space $L(n, *)$. A strong inversion on $K$ is a $\pi$-rotation of $S^{3}$ which leaves $K$ invariant and has axis meeting $K$ at exactly two points. Finally, in this paper the terms cover and covering always mean nontrivial ones (i.e., of degrees greater than 1).

The first lemma reduces coverings between knot complements to cyclic coverings.

LEMMA 1. If $p: E(K) \rightarrow E\left(K_{1}\right)$ is a covering between knot complements, then $p$ is a cyclic covering.

Proof. This is Theorem 1.1 of [GW].

LEMMA 2. Suppose $f$ is a nontrivial (i.e. not the identity) periodic map on a torus $T$. Then $f$ is isotopic to the identity if and only if $f$ is fixed point free and orientation preserving.

Proof. Let $A_{f}$ be the induced matrix of $f$ on $H_{1}(T)$. By 12.4 of [H], under a suitable basis of $H_{1}(T), A_{f}$ is one of the seven matrices on p. 123 of $[\mathbf{H}]$. If $f$ is fixed point free and orientation preserving, then we have $\operatorname{det} A_{f}=1$, and the Lefschetz number $L(f)=2$ $\operatorname{tr} A_{f}=0$. These conditions force $A_{f}$ to be the identity matrix. Hence $f$ is isotopic to the identity. 
Conversely, if $f$ is isotopic to the identity, then $f$ is orientation preserving and $L(f)=0$. Then it is an elementary fact that $f$ is the identity or is fixed point free.

The next lemma is quoted from [W1]. It builds up a connection between coverings of knot complements and cyclic surgeries on knots. The first part is also in [GW] with somewhat different proof.

LEMMA 3. Let $K$ be a nontrivial knot.

(1) If $E(K)$ is $n$-fold cyclic covered by $E(\widetilde{K})$ for some knot $\widetilde{K}$, then $\pi_{1}(K, n / r)=\mathbf{Z}_{n}$.

(2) If $(K, n / r)=L(n)$, then $E(K)$ is $n$-fold cyclic covered by $E(\widetilde{K})$ for some knot $\widetilde{K}$.

(3) If $K$ is not a torus knot, then $r=1$ in (1) and (2).

Proof. (1) If $E(\widetilde{K}) \rightarrow E(K)$ is an $n$-fold cyclic covering, then the action of deck transformation group $\Pi=\left(\tau \mid \tau^{n}=1\right)$ on $E(\tilde{k})$ is orientation preserving and fixed point free. Let $T=\partial(N(\widetilde{K}))$. Then $\left.\tau\right|_{T}$, the restriction of $\tau$ on $T$, is an orientation preserving and fixed point free periodic map. By Lemma $2,\left.\tau\right|_{T}$ is isotopic to the identity. In particular it sends meridian to meridian. So we can use the standard disk extention to extend $\left.\tau\right|_{T}$ over the solid torus $N(\tilde{k})$ in a periodic way, getting an action of $\Pi$ on $S^{3}$ which has no fixed point in $S^{3}-\widetilde{K}$. As the action is cyclic and orientation preserving, the fixed point set of $\Pi$ is either $\widetilde{K}$ or the empty set. Now $\widetilde{K}$ is knotted because $K$ is, so by Smith Conjecture it cannot be the fixed point set of $\Pi$. Hence $\Pi$ acts freely on $S^{3}=E(\widetilde{K}) \cup N(\widetilde{K})$, and

$$
S^{3} / \Pi=(E(\widetilde{K}) / \Pi) \cup(N(\widetilde{K}) / \Pi)=E(K) \cup\left(S^{1} \times D^{2}\right)=(K, n / r)
$$

where the surgery coefficient is of the form $n / r$ because $\Pi=\mathbf{Z}_{n}$.

(2) If $(K, n / r)=L(n)$, then we have an $n$-fold cyclic covering $q: S^{3} \rightarrow L(N)$. the meridian $m$ of $K$ represents a generator of $H_{1}((K, n / r))=\pi_{1}((K, n / r))=\mathbf{Z}_{n}$; so $q^{-1}(m)$ is connected. It implies that $q^{-1}(N(K))$ is a single solid torus. Let $\widetilde{K}$ be the central curve of $q^{-1}(N(K))$. Then $q^{-1}(N(K))$ is a regular neighborhood of $\widetilde{K}$, and $q$ restricting to $E(\widetilde{K})=S^{3}-q^{-1}(N(K))$ is a cyclic covering onto $E(K)$.

(3) follows from [CGLS].

By a hyperbolic structure (or metric) we mean one which is complete and has finite volume. A hyperbolic 3-manifold $M$ is a manifold 
whose interior admits such a hyperbolic structure. We use $M_{\rho}$ to denote Int $M$ together with a given hyperbolic metric $\rho$.

LEMMA 4. Let $h$ be a map on a hyperbolic 3-manifold $M_{\rho}$. Then the following two statements are equivalent:

(1) $h$ is conjugate to an isometry on $M_{\rho}$;

(2) there exists a hyperbolic metric $\rho_{1}$ on $M$ under which $h$ is an isometry.

Proof. Suppose $g$ is a self-homeomorphism of $M$ such that $g h g^{-1}$ is an isometry on $M_{\rho}$. Let $\rho_{1}=g^{*} \rho$ be defined by $\rho_{1}(x, y)=$ $\rho(g(x), g(y))$ for all $x, y \in M$. Then we have

$$
\begin{aligned}
\rho_{1}(h(x), h(y)) & =\rho(g h(x) k, g h(y))=\rho\left(g h g^{-1} g(x), g h g^{-1} g(y)\right) \\
& =\rho(g(x), g(y))=\rho_{1}(x, y) ;
\end{aligned}
$$

i.e., $h$ is an isometry on $M_{\rho_{1}}$.

Conversely, suppose (2) is true. By Mostow's Rigidity Theorem [T, 5.7.2], id: $\pi_{1}\left(M_{\rho}\right) \rightarrow \pi_{1}\left(M_{\rho_{1}}\right)$ can be realized by a unique isometry $g: M_{\rho} \rightarrow M_{\rho_{1}}$. So we have

$$
\begin{aligned}
\rho(x, y) & =\rho_{1}(g(x), g(y))=\rho_{1}(h g(x), h g(y)) \\
& =\rho\left(g^{-1} h g(x), g^{-1} h g(y)\right) ;
\end{aligned}
$$

i.e., $g^{-1} h g$ is an isometry on $M_{\rho}$.

The next two lemmas are used to prove Theorem 1 for hyperbolic knots.

Lemma 5. Suppose $E=E(K)$ is hyperbolic, and $\Pi_{1}=\left(\tau_{1} \mid \tau_{1}^{p}=1\right)$ and $\Pi_{2}=\left(\tau_{2} \mid \tau_{2}^{q}=1\right)$ act freely on $E$. Then there is a hyperbolic metric $\rho$ on Int $E$ and a homeomorphism $g$ on $E$ such that $G=$ $\left\langle\tau_{1}, g \tau_{2} g^{-1}\right\rangle$ is a finite abelian group.

Proof. Let $p_{i}: E \rightarrow E / \Pi_{i}$ be the covering map. Since $E$ is hyperbolic, $E / \Pi_{i}$ is homotopic to a hyperbolic manifold [T, 6.7.3]. Since $E / \Pi_{i}$ is a $P^{2}$-irreducible 3 -manifold (by [MSY, Theorem 3]) with a torus boundary component, it is a Haken manifold. So $E / \Pi_{i}$ itself admits a hyperbolic structure. For any given hyperbolic metric $\bar{\rho}$ on $E / \Pi_{i}$, let $\rho_{i}=p_{i}^{*} \bar{\rho}$ be the pullback metric on $E$. Then $\tau_{i}$ is an isometry on $E_{\rho_{i}}$.

Now fix a metric $\rho_{1}$ on $E$ under which $\tau_{1}$ is an isometry. We have just shown that $\tau_{2}$ is an isometry under certain hyperbolic metric. 
So by Lemma 4 it is conjugate to an isometry with respect to $\rho_{1}$; i.e., there is a homeomorphism $g$ such that $g \tau_{2} g^{-1}$ is an isometry on $E_{\rho_{1}}$. Thus $G=\left\langle\tau_{1}, g \tau_{2} g^{-1}\right\rangle$ is a group of isometries. By the Mostow-Thurston Theorem [T, 5.7.4], $G$ is a finite group.

It remains to show that $G$ is abelian. Let $E_{\varepsilon}$ be the submanifold of Int $E$ consisting of points contained in some hyperbolic ball of radius $\varepsilon$. Choose $\varepsilon$ small enough so that $E_{\varepsilon}$ is homeomorphic to $E$ (see 5.10 .2 and 5.11 .1 of $[\mathrm{T}]$ ). Since the elements of $G$ are isometries on $E, E_{\varepsilon}$ is invariant under the $G$ action. Especially, $T=\partial E_{\varepsilon}$ is invariant under the $G$ action. As $\left.\tau_{1}\right|_{T}$ and $\left.\tau_{2}\right|_{T}$ are fixed point free orientation preserving periodic maps on $T$, they both induce the identity map on $\pi_{1}(T)$ by Lemma 2 . Thus every element in $G$ induces identity on $\pi_{1}(T)$. Again by Lemma 2, except the identity, every element in $\left.G\right|_{T}$ is a fixed point free orientation preserving periodic map. In other words, $\left.G\right|_{T}$ is a free action. So we can consider $T$ as a covering space over $T /\left(\left.G\right|_{T}\right)$, and $\left.G\right|_{T}$ as the covering transformation group. Since $T /\left(\left.G\right|_{T}\right)$ is a torus, the quotient group $\left.G\right|_{T}=\pi_{1}\left(T /\left(\left.G\right|_{T}\right)\right) / \pi_{1}(T)$ is abelian.

LEMMA 6. If a non-torus knot $K$ admits a symmetry which is not a strong inversion, then $E(K)$ cannot be covered by a knot complement.

Proof. In [WZ], Wang and Zhou proved that if a non-torus knot $K$ admits a symmetry which is not a strong inversion, then there is no cyclic surgery on $K$. Lemma 6 then follows from Lemma 3(2).

We use $K(p, q)$ to denote the $(p, q)$-torus knot, and $K(p, q ; r, s)$ to denote the $(r, s)$-cable of $K(p, q)$. Without loss of generality we may assume $p>q>0$ (otherwise change the orientation of $S^{3}$ ). The next two lemmas are used to prove the theorem for satellite knots. Recall that $(K, n)$ denotes the manifold obtained from $S^{3}$ by Dehn surgery on $K$ with slope $n$.

Lemma 7. Suppose $K$ is a satellite knot. Then $(K, n)$ is a lens space if and only if $K=K(p, q ; 2,2 p q \pm 1)$ and $n=4 p q \pm 1$.

Proof. This is a result of Bleiler-Litherland [BL] and $\mathrm{Wu}[\mathbf{W u}]$.

Let $M$ be the complement of $K(p, q ; 2,2 p q+1)$, let $N$ be the complement of $K(p, q ; 2,2 p q-1)$. Denote by $\widetilde{M}$ the $4 p q+1$ fold cyclic covering of $M$, and by $\widetilde{N}$ the $4 p q-1$ fold covering of $N$. 


\section{LeMMA 8. $\widetilde{M}$ is not homeomorphic to $\widetilde{N}$.}

Proof. Let $T$ be a torus which divides $M$ into two maximal Seifert pieces $M_{1}$ and $M_{2}$, where $M_{1}=E(k(p, q))$, and $M_{2}$ is a cable space $C(2,2 p q+1)$ (see e.g. [G] for definition of cable spaces). Consider $T$ as the boundary of the knot complement $M_{1}$. Choose a pair of simple closed curves $m$ and $l$ on $T$ so that they represent the homology of the standard meridian-longitude pair of $K(p, q)$ (rather than that of $K(p, q ; 2,2 p q+1))$.

Denote by $\tilde{m}, \tilde{l}, \widetilde{M}_{1}, \widetilde{M}_{2}, \widetilde{T}$ the lifting (preimage) of $m, l, M_{1}$, $M_{2}, T$, respectively. It is easy to show that if $c$ is a simple closed curve in $M$ representing an element $k$ in $\mathrm{Z}=H_{1} M$, then its lifting $\tilde{c}$ in an $n$-fold cyclic covering $\widetilde{M}$ of $M$ has $\operatorname{gcd}(k, n)$ components. Since homologically $m$ is 2 times a meridian of $K(p, q ; 2,2 p q+1)$ and since 2 is coprime with $4 p q+1, \tilde{m}$ is connected. Especially, $\widetilde{M}_{1}, \widetilde{M}_{2}, \widetilde{T}$ are all connected. Since $l$ is null-homologous, $\tilde{l}$ consists of $4 p q+1$ parallel simple closed curves. Let $l^{\prime}$ be one of these curves. Then $[\tilde{l}]=(4 p q+1)\left[l^{\prime}\right]$. Note that $\tilde{m}$ intersects $\tilde{l}$ transversely at $4 p q+1$ points (because $m$ intersects $l$ at one point), so $\tilde{m}$ intersects $l^{\prime}$ exactly once. Thus the simple closed curves $\tilde{m}$ and $l^{\prime}$ generate $H_{1}(\widetilde{T})$.

It is well known that Seifert fibrations of both $M_{1}$ and $M_{2}$ are unique up to isotopy (see Theorem 3.9 of [S] and its refinement). Let $\alpha_{i}$ be the simple closed curve on $T$ representing a fiber of $M_{i}$. One can check that $\left[\alpha_{1}\right]=p q[m]+[l]$, and $\left[\alpha_{2}\right]=(2 p q+1)[m]+2[l]$. Let $\tilde{\alpha}_{i}$ be the lift of $\alpha_{i}$. Since both $p q$ and $2 p q+1$ are coprime with $4 p q+1$, the $\tilde{\alpha}_{i}$ 's are connected; so each $\tilde{\alpha}_{i}$ is a fiber of the lifted fibration of $\widetilde{M}_{i}$. We have

$$
\begin{aligned}
& {\left[\tilde{\alpha}_{1}\right]=p q[\tilde{m}]+[\tilde{l}]=p q[\tilde{m}]+(4 p q+1)\left[\tilde{l}^{\prime}\right]} \\
& {\left[\tilde{\alpha}_{2}\right]=(2 p q+1)[\tilde{m}]+2[\tilde{l}]=(2 p q+1)[\tilde{m}]+2(4 p q+1)\left[l^{\prime}\right]}
\end{aligned}
$$

Since $[\tilde{m}]$, $\left[\tilde{l}^{\prime}\right]$ generates $H_{1}(T)$, we can compute the intersection number of $\left[\tilde{\alpha}_{1}\right]$ and $\left[\tilde{\alpha}_{2}\right]$ by

$$
\left[\tilde{\alpha}_{1}\right]\left[\tilde{\alpha}_{2}\right]=\left|\operatorname{det}\left(\begin{array}{cc}
p q & 4 p q+1 \\
2 p q+1 & 8 p q+2
\end{array}\right)\right|=4 p q+1 .
$$

Construct in a similar way a decomposing torus $\widetilde{T}^{\prime}$ which divides $\widetilde{N}$ into $\widetilde{N}_{1}$ and $\widetilde{N}_{2}$, and simple closed curves $\tilde{\beta}_{1}, \tilde{\beta}_{2}$ which represent fibers of $\tilde{n}_{1}$ and $\tilde{N}_{2}$ respectively. By a similar computation we have $\left[\tilde{\beta}_{1}\right]\left[\tilde{\beta}_{2}\right]=4 p q-1$. 
If $\widetilde{M}$ and $\widetilde{N}$ are homeomorphic, by the Torus Decomposition Theorem of Jaco-Shalen-Johannson (see $[\mathbf{J}]$ ) and the uniqueness of fibration of $\widetilde{M}_{i}$ (see e.g. [J, Theorem VI.18]), there is a homeomorphism $\varphi: \widetilde{M} \rightarrow \widetilde{N}$ such that $\varphi(\widetilde{T})=\widetilde{T}^{\prime}$ and $\varphi\left(\tilde{\alpha}_{i}\right)=\tilde{\beta}_{i}, i=1$, 2, so $\left[\tilde{\alpha}_{1}\right]\left[\tilde{\alpha}_{2}\right]=\left[\tilde{\beta}_{1}\right]\left[\tilde{\beta}_{2}\right]$. But this contradicts the above computation.

Lemma 9. Suppose $E\left(K_{1}\right) \rightarrow E\left(K_{2}\right)$ is a cyclic covering between knot complements. If one of the $K_{i}$ is a torus knot $K(p, q)$, then both $E\left(K_{1}\right)$ and $E\left(K_{2}\right)$ are homeomorphic to $E(K(p, q))$, and the covering degree is rpq \pm 1 for some $r$.

Proof. If $K_{i}$ is a torus knot, then it is a Seifert manifold. Hence both $E\left(K_{1}\right)$ and $E\left(K_{2}\right)$ are Seifert manifolds. It implies that both $K_{1}$ and $K_{2}$ are torus knots (see [J, IX 22]). So we may assume $K_{2}=K(p, q)$.

By the work of Moser [M] $\pi_{1}(k(p, q), n / r)$ is cyclic if and only if $n=r p q \pm 1$. Thus $E\left(K_{1}\right)$ is an $r p q \pm 1$ fold cyclic covering of $E(k(p, q))$. But such a covering space is homeomorphic to $E(K(p, q))$, because $T(p, q)$ is a fibered knot with holonomy a period map of order $p q$ (see e.g. [R]).

With Lemma 9 in hand, we need only to prove Theorem 1 for hyperbolic knots and satellite knots.

Proof of Theorem 1 for hyperbolic knots. Suppose the complement $E(K)$ of a hyperbolic knot $K$ covers two knot complements $E\left(K_{1}\right)$ and $E\left(K_{2}\right)$. By Lemma 1 , these coverings are cyclic. Let $\Pi_{1}=$ $\left(\tau_{1} \mid \tau_{1}^{p}=1\right)$ and $\Pi_{2}=\left(\tau_{2} \mid \tau_{2}^{q}=1\right)$ be the deck transformation groups. For any homeomorphism $g$ of $E(K), E(K) /\left\langle\tau_{i}\right\rangle$ is homeomorphic to $E(K) /\left\langle g \tau_{i} g^{-1}\right\rangle$. So by Lemma 5, after replacing $\tau_{2}$ by some $g \tau_{2} g^{-1}$ if necessary, we may assume that $\tau_{1}, \tau_{2}$ and the hyperbolic metric on $E(K)$ have been chosen so that the action of $G=\left\langle\tau_{1}, \tau_{2}\right\rangle$ is the action of a finite abelian group.

Claim. $G=\left\langle\tau_{1}\right\rangle=\left\langle\tau_{2}\right\rangle$.

Otherwise, suppose $\left\langle\tau_{1}\right\rangle=\left\langle\tau_{2}\right\rangle$, say, is a proper subgroup of $G$. As $G$ is abelian, $\left\langle\tau_{1}\right\rangle$ is a normal subgroup; so the non-trivial group $G /\left\langle\tau_{1}\right\rangle$ acts on $E(K)\left\langle\tau_{1}\right\rangle=E\left(K_{1}\right)$. Since the restriction of $G$ on $\partial E(K)$ is a free action, it follows from covering space theory that the restriction of $G /\left\langle\tau_{1}\right\rangle$ on $\partial E\left(K_{1}\right)$ is a free action; so the action of 
$G /\left\langle\tau_{1}\right\rangle$ on $E\left(K_{1}\right)$ is not a strong inversion. By Lemma $3 E\left(K_{1}\right)$ admits a cyclic surgery, but by Lemma 6 it cannot admit such a surgery, a contradiction. So we have $G=\left\langle\tau_{1}\right\rangle=\left\langle\tau_{2}\right\rangle$.

Therefore, $E\left(K_{1}\right)$ and $E\left(K_{2}\right)$ are homeomorphic.

Proof of Theorem 1 for satellite knots. Suppose the complement $E(K)$ of a satellite knot $K$ covers two non-homeomorphic knot complements $E\left(K_{1}\right)$ and $E\left(K_{2}\right)$, with degree $n_{1}$ and $n_{2}$ respectively. By Lemma 3, $E\left(K_{i}\right)$ admits a cyclic surgery with coefficient $n_{i} / r_{i}$. By Lemma 7, we must have $K_{i}=K\left(p_{i}, q_{i} ; 2,2 p_{i} q_{i}+\varepsilon_{i}\right)$ and $n_{i}=$ $4 p_{i} q_{i}+\varepsilon_{i}$, where $\varepsilon_{i}= \pm 1$; so $E(K)$ is a $4 p_{i} q_{i}+\varepsilon_{i}$ fold cyclic covering of $E\left(K_{i}\right)$. Use the notations in the proof of Lemma 8, and write $E\left(K_{i}\right)=M_{1}^{(i)} \cup M_{1}^{(i)}=E\left(K\left(p_{i}, q_{i}\right)\right) \cup C\left(2,2 p_{i} q_{1}+\varepsilon_{i}\right)$. It was implied in the proofs of Lemmas 8 and 9 that the preimage $\widetilde{M}_{1}^{(i)}$ of $M_{1}^{(i)}$ in $E(K)$ is homeomorphic to $M_{1}^{(i)}$ itself, while by the uniqueness of torus decomposition of $E(K)$ we must have $\widetilde{M}_{1}^{(1)} \cong$ $\widetilde{M}_{1}^{(2)}$. Therefore, $E\left(K\left(p_{1}, q_{1}\right)\right) \cong \widetilde{M}_{1}^{(1)} \cong \tilde{m}_{1}^{(2)} \cong E\left(K\left(p_{2}, q_{2}\right)\right)$. It is well known that torus knots are determined by their complements, so we have $\left(p_{1}, q_{1}\right)=\left(p_{2}, q_{2}\right)=(p, q)$ for some $p, q$. Now if $K_{1}=K\left(p, q ; 2,2 p q+\varepsilon_{1}\right)$ and $K_{2}=K\left(p, q ; 2,2 p q+\varepsilon_{2}\right)$ are not the same, then $\varepsilon_{1}=-\varepsilon_{2}$, and $E(K)$ is a $4 p q+\varepsilon$ fold covering of $K(p, q ; 2,2 p q+\varepsilon)$ for both $\varepsilon= \pm 1$, which is impossible by Lemma 8.

\section{REFERENCES}

[BL] S. Bleiler and R. Litherland, Lens space and Dehn surgery, Proc. Amer. Math. Soc., 107 (1989), 1127-1131.

[BM] H. Bass and J. Morgan, The Smith Conjecture, Academic Press, (1984).

[CGLS] M. Culler, C. Gordon, J. Luecke and P. Shalen, Dehn surgery on knots, Ann. of Math., 125 (1987), 237-300.

[GW] F. Gonzales-Acuna and W. Whitten, Imbedding knot groups in knot groups, Lecture Notes in Pure and Appl. Math., vol. 105 (1987), 147-156.

[G] C. Gordon, Dehn surgery and satellite knots, Trans. Amer. Math. Soc., 275 (1983), 687-708.

[H] J. Hemple, 3-manifold, Ann. of Math Studies, vol. 86, Princeton University Press, Princeton, NJ, 1976.

[J] W. Jaco, Lectures on three-manifold topology, Regional Conference Series in Mathematics, 43 (1981).

[MSY] W. Meeks, L. Simon and S. Yau, Embedded minimal surfaces, exotic spheres, and manifolds with positive Ricci curvature, Ann. of Math., 116 (1982), 621659.

[M] L. Moser, Elementary surgery along torus knots, Pacific J. Math., 38 (1971), 734-745. 
[R] D. Rolfsen, Knots and Links, Publish or Perish, (1976).

[S] P. Scott, The geometry of 3-manifolds, Bull. London Math. Soc., 15 (1983), 401-478.

[T] W. Thurston, The geometry and topology of 3-manifolds, Princeton University (1978).

[W1] S. Wang, thesis, UCLA, (1988).

[W2] _ Cyclic surgery on knots, Proc. Amer. Math. Soc., 107 (1989), 10911094.

[W3] _ 3-manifolds covers only finitely many 3-manifolds, Quart. J. Math. Oxford, 42 (1991), 113-124.

[WZ] S. Wang and Q. Zhou, Symmetry and cyclic surgery on knots, Trans. Amer. Math. Soc., 330 (1992), 665-676.

[Wu] Y.-Q. Wu, Cyclic surgery and satellite knots, Topology Appl., 36 (1990), 205-208.

Received June 5, 1991 and in revised form February 14, 1992.

Peking University

BEIJING 100871

The People's Republic of China

AND

UNIVERSITY OF CALIFORNIA

SANTA BARbara, CA 93106 



\title{
PACIFIC JOURNAL OF MATHEMATICS \\ Founded by \\ E. F. BECKENBACH (1906-1982) F. WolF (1904-1989)
}

\section{EDITORS}

\section{S. VARADARAJAN \\ (Managing Editor) \\ University of California \\ Los Angeles, CA 90024-1555 \\ vsv@math.ucla.edu}

\section{F. Michael Christ}

University of California

Los Angeles, CA 90024-1555

christ@math.ucla.edu

\section{Herbert Clemens}

University of Utah

Salt Lake City, UT 84112

clemens@math.utah.edu

\author{
THOMAs ENRIGHT \\ University of California, San Diego \\ La Jolla, CA 92093 \\ tenright@ucsd.edu \\ Nicholas ERcolani \\ University of Arizona \\ Tucson, AZ 85721 \\ ercolani@math.arizona.edu \\ R. FINN \\ Stanford University \\ Stanford, CA 94305 \\ finn@gauss.stanford.edu \\ VAUghan F. R. JoNeS \\ University of California \\ Berkeley, CA 94720 \\ vfr@math.berkeley.edu
}

\section{SUPPORTING INSTITUTIONS}

UNIVERSITY OF ARIZONA

UNIVERSITY OF BRITISH COLUMBIA

CALIFORNIA INSTITUTE OF TECHNOLOGY

UNIVERSITY OF CALIFORNIA

UNIVERSITY OF MONTANA

UNIVERSITY OF NEVADA, RENO

NEW MEXICO STATE UNIVERSITY OREGON STATE UNIVERSITY

\author{
UNIVERSITY OF OREGON \\ UNIVERSITY OF SOUTHERN CALIFORNIA \\ STANFORD UNIVERSITY \\ UNIVERSITY OF HAWAII \\ UNIVERSITY OF UTAH \\ WASHINGTON STATE UNIVERSITY \\ UNIVERSITY OF WASHINGTON
}

The Supporting Institutions listed above contribute to the cost of publication of this Journal, but they are not owners or publishers and have no responsibility for its content or policies.

Mathematical papers intended for publication in the Pacific Journal of Mathematics should be in typed form or offset-reproduced (not dittoed), double spaced with large margins. Please do not use built up fractions in the text of the manuscript. However, you may use them in the displayed equations. Underline Greek letters in red, German in green, and script in blue. The first paragraph must be capable of being used separately as a synopsis of the entire paper. In particular it should contain no bibliographic references. Please propose a heading for the odd numbered pages of less than 35 characters. Manuscripts, in triplicate, may be sent to any one of the editors. Please classify according to the 1991 Mathematics Subject Classification scheme which can be found in the December index volumes of Mathematical Reviews. Supply name and address of author to whom proofs should be sent. All other communications should be addressed to the managing editor, or Julie Speckart, University of California, Los Angeles, California 90024-1555.

There are page-charges associated with articles appearing in the Pacific Journal of Mathematics. These charges are expected to be paid by the author's University, Government Agency or Company. If the author or authors do not have access to such Institutional support these charges are waived. Single authors will receive 75 free reprints; joint authors will receive a total of 100 free reprints. Additional copies may be obtained at cost in multiples of 50 .

The Pacific Journal of Mathematics (ISSN 0030-8730) is published monthly except for July and August. Regular subscription rate: $\$ 200.00$ a year (10 issues). Special rate: $\$ 100.00$ a year to individual members of supporting institutions.

Subscriptions, orders for numbers issued in the last three calendar years, and changes of address should be sent to Pacific Journal of Mathematics, P.O. Box 969, Carmel Valley, CA 93924, U.S.A. Old back numbers obtainable from Kraus Periodicals Co., Route 100, Millwood, NY 10546.

The Pacific Journal of Mathematics at P.O. Box 969, Carmel Valley, CA 93924 (ISSN 0030-8730) is published monthly except for July and August. Second-class postage paid at Carmel Valley, California 93924, and additional mailing offices. Postmaster: send address changes to Pacific Journal of Mathematics, P.O. Box 969, Carmel Valley, CA 93924.

PUBLISHED BY PACIFIC JOURNAL OF MATHEMATICS, A NON-PROFIT CORPORATION

This publication was typeset using $\mathcal{A} \mathcal{S} S-\mathrm{T}_{\mathrm{E}} \mathrm{X}$, the American Mathematical Society's $\mathrm{T}_{\mathrm{E}} \mathrm{X}$ macro system.

Copyright (c) 1993 by Pacific Journal of Mathematics 


\section{PACIFIC JOURNAL OF MATHEMATICS}

Volume $158 \quad$ No. $2 \quad$ April 1993

On the extension of Lipschitz functions from boundaries of subvarieties to

201 strongly pseudoconvex domains

K. ADACHI and HiRoshi KAJIMOTO

On a nonlinear equation related to the geometry of the diffeomorphism group 223

DaVid DaI-WAi BaO, JACQUES LAFONTAINE and TUdOR S. RATIU

Fixed points of boundary-preserving maps of surfaces

ROBERT F. BROWN and BRIAN SANDERSON

On orthomorphisms between von Neumann preduals and a problem of Araki 265

L. J. BUNCE and JOHN DAVID MAITLAND WRIGHT

Primitive subalgebras of complex Lie algebras. I. Primitive subalgebras of 273 the classical complex Lie algebras

\section{V. CHEKALOV}

$L^{n}$ solutions of the stationary and nonstationary Navier-Stokes equations in 293 $R^{n}$

\section{ZHI MiN CHEN}

Some applications of Bell's theorem to weakly pseudoconvex domains

XiAO JUN HUANG

On isotropic submanifolds and evolution of quasicaustics

STANISŁAW JANECZKO

Currents, metrics and Moishezon manifolds

SHANYU JI

Stationary surfaces in Minkowski spaces. I. A representation formula

JIANGFAN LI

The dual pair $(U(1), U(1))$ over a $p$-adic field

Courtney Hughes Moen

Any knot complement covers at most one knot complement 